\title{
Investigating Learners' Attitudes Toward Virtual Reality Learning Environments in Embryology Education
}

\author{
Salsabeel F. M. Alfalah ${ }^{1}$, Jannat F. Falah ${ }^{2}$, Nadia Muhaidat ${ }^{3}$, Mutasem Elfalah ${ }^{4} \&$ Orwa Falah $^{5}$ \\ ${ }^{1}$ Department of Computer Information Systems, King Abdullah II School of Information Technology, The \\ University of Jordan, Jordan \\ ${ }^{2}$ Department of Computer Science, Faculty of Information Technology, Al-Balqa`Applied University, Jordan \\ ${ }^{3}$ Department of Obstetrics and Gynecology, Faculty of Medicine, The University of Jordan, Jordan \\ ${ }^{4}$ Department of Special Surgery Ophthalmology Unit, Faculty of Medicine, The University of Jordan, Jordan \\ ${ }^{5}$ Department of Vascular Surgery, Faculty of Medicine, The University of Edinburgh, UK \\ Correspondence: Salsabeel F. M. Alfalah, King Abdullah II School of Information Technology, The University of \\ Jordan, Queen Rania Street, Amman 11942, Jordan. Tel: 962-653-550-00 Ext: 22562. E-mail: \\ Salsabeel.alfalah@gmail.com
}

Received: Oct. 11, 2018

doi:10.5539/mas.v13n1p57

\author{
Accepted: Nov. 1, 2018 Online Published: December 10, 2018 \\ URL: https://doi.org/10.5539/mas.v13n1p57
}

\begin{abstract}
Embryology is taught to undergraduates in a variety of health sciences, largely depending on traditional teaching methods. The human embryo's development is complex and dynamic, and to digest all the related facts, students are always searching for educational tools to enhance their learning experience. Virtual Reality (VR) is a promising technology that aids in the realization of 3 Dimensional (3D) relations of structures by visualization rather than memorization. This paper utilizes a mixed method research consisting of focus group interviews, followed by a questionnaire to evaluate the current teaching methodology, identify shortfalls of traditional learning tools, and determine how VR may enhance the learning process, and the students' acceptance of introduction of this technology into their curriculum. It demonstrates that a VR embryology system would provide a valuable addition to existing educational tools, which is appealing to users, and has the potential to overcome some of the current methods' shortfalls.
\end{abstract}

Keywords: virtual reality, technology, student assessment, medical education, 3D, embryology

\section{Introduction}

The science of embryology has always been a crucial component of health science faculties' curricula. Embryology is usually taught as part of the anatomy course to undergraduate health sciences students, such as medicine, dentistry, nursing, amongst others. Learning about the development of the human embryo, and the rapid changes it undergoes in the early stages of intrauterine life is of paramount importance, not just to understand the origin of the different parts of the human body, and the stages through which each part passes in order to reach its final structure, but also because it provides insight into how this process can be influenced by medical interventions, such as assisted reproduction techniques, as well as more understanding of congenital abnormalities and diseases (Carlson, 2002; Drake, Lowrie \& Prewitt, 2002).

The understanding of the aforementioned requires understanding of the three dimensional structure of the embryo, as well as the changes it undergoes over time (Carlson, 2002). Several studies have demonstrated that the utilization of novel technology as teaching aids for this particular purpose can be of significant benefit for both teachers and students. (Dimitropoulos, Manitsaris, \& Mavridis, 2007; Falah, Harrison, Charissis, \& Wood, 2013; Falah et al., 2014; Ginani, Gadelha, \& Augusto, 2012).

Despite the fact that the aforementioned points provide encouragement for the utilization of novel technology in education, there remains the issue of user acceptance, without which implementation of the proposed changes to the learning process will not achieve the desired potential. It is therefore of utmost importance that the users willingness to use the technology is assessed prior to developing a new system (Dillon, 2001; Sánchez, Barreiro, \& Maojo, 2000; Venkatesh, Speier, \& Morris, 2002). If virtual reality is planned to be used as an educational 
medium, students should perceive this to be useful and accept it as a valuable addition to the learning process (Alfalah, 2018). For this reason the perception of the users, namely the students studying the embryology course towards VR is evaluated as part of this research.

\section{Background}

Embryology, the science of the origin and development of the individual from fertilization of an oocyte to the end of the eighth week of development and, by extension, during any stage of prenatal development (Dorland, 2007), is usually represented in the medical school curriculum as part of the basic sciences taught in the first few years of undergraduate studies, and is usually part of the anatomy course.

Studies have revealed that students that perceive embryology as a valuable part of their undergraduate studies do so because they think that allows them to improve clinical management and understanding (Scott, Charles, \& Holland, 2013). Apart from understanding the developmental origins of the diverse anatomy of the human body, its coverage of a number of clinical subjects, such as the basis of gametogenesis and fertilization, congenital anomalies, and the effect of intrauterine events such as congenital infection on the development of the fetus, which are of obvious relevance to number of specialties such as pediatrics and obstetrics and gynecology, makes embryology a vital branch of knowledge for anyone planning a future career in the field of health and medicine.

There are several views of what an embryology curriculum should include. One approach is to divide the curriculum into three main sections according to the stage of development, starting with gametogenesis, transportation, fertilization and fusion of the male and female genetic material, moving to the sequence of the structural development of the different organ systems in the human body, in addition to defining the time at which these events occur (Carlson, 2002).

Traditionally, this subject has been taught in ways similar to those used for gross anatomy teaching, depending heavily on textbooks and lectures, with the addition of traditional teaching aids such as $2 \mathrm{D}$ images, models, and in some cases preserved human embryos. Changes in medical curricula have led to a reduction in the amount of time available to the students during which they are exposed to learning tools (Patel \& Moxham, 2008). The majority of embryology courses are taught as part of an integrated program, and there seems to be a trend towards a reduction in laboratory exposure during the course (Drake, McBride, Lachman, \& Pawlina, 2009). Nowadays, most medical schools provide a lecture-based course, with no laboratory time at all (Carlson, 2002). Currently at the University of Jordan, this subject is taught to medical and dentistry students as part of the basic sciences curriculum in first or second year, and the curriculum comprises of a number of lectures without any allocated laboratory sessions.

As the understanding of embryological development requires the student not only to study the three dimensional structures of the embryo, and understand the spatial relations between the different parts, but also to realize the evolution of these structures over the course of time, in a complex, dynamic process (Carlson, 2002; Moraes, \& Pereira, 2010), the student has to rely heavily on their imagination to appreciate and visualize the structural changes that the embryo goes through.

Recently a number of studies have evaluated the role of more novel teaching methods, in particular multimedia approaches, to enhance the educational process. This has shown the potential to solve some problems associated with the medical curriculum, such as reducing the gap between basic sciences and clinical training (Moraes \& Pereira, 2010). Virtual reality technology, in particular, is proving to be very useful for the 3D visualization and of structures as well as providing a medium in which the user can interact with the material through a number of functionalities while being immersed into a computer-generated virtual environment (Dimitropoulos, Manitsaris, \& Mavridis, 2008). Morphing techniques are used to generate animations of structures that evolve over time by averaging between structures at different time points. These time points should not be too distant in order not to lose important milestones along the way; however generating animations in this way can be extremely useful to aid students in understanding the developmental process (Carlson, 2002; Yamada et al., 2006).

Yamada et al. developed a series of 3D models of the different stages of development of the human embryo and videos that demonstrate the evolution of the embryo from one stage to the next, using magnetic resonance microscopy images with the aid of computer graphics, and utilizing the morphing technique to connect the different stages in the videos (Yamada et al., 2006).

Anatomy teaching, of which embryology is a subdivision has greatly benefited from the emerging virtual reality technology in recent years. Several VR systems for teaching the anatomy of various body parts such as the heart (Falah et al., 2015) and the ear (Nicholson, Chalk, Funnell, \& Daniel, 2006) have been developed, and some universities have even established virtual anatomy laboratories within educational multiuser virtual environments, 
specifically Second LifeTM, to provide a virtual space where students can learn and interact (Richardson, Hazzard, Challman, Morgenstein, \& Brueckner, 2011).

In addition to the facilitation of novel techniques as teaching aids, there is also a trend of moving towards webbased learning. This provides online access for the students to the course material as well as some complementary tools such as images and illustrations. The availability of animations however, which are particularly useful for understanding the developmental events in embryology, is still very limited in online course materials, due to complexity and cost (Carlson, 2002).

This study aims to evaluate the current teaching methods used to teach embryology, the adequacy of those methods for achieving the learning goals of the students, and to identify gaps and shortfalls that could potentially be improved by introducing novel technology, namely virtual reality, as a learning and teaching aid.

In addition to the aforementioned objectives, this study also examines the attitude of students towards the concept of introducing a novel technology, in particular VR, as a learning aid for their embryology curriculum. The new generation of undergraduate students is accustomed to the use of Information Technology (IT) in all aspects of their life, and there is growing demand for the educational methods to catch up with the technological innovations that are becoming available to this generation (Alfalah, S., Falah, J., Alfalah, T., Elfalah \& Falah, O., 2017; Kron, Gjerde, Sen \& Fetters, 2010). For such a development to be successful, it has to be met with acceptance from the proposed users (Alfalah, 2018; Sánchez et al., 2000; Dillon, 2001; Venkatesh et al., 2002). Therefore, the students' perceptions towards virtual reality, their willingness to use such technology, and whether they consider its introduction into the learning process to be of value to them, is a major determinant of the success of the proposed addition to the embryology course, namely the use of a Virtual Reality system to aid the understanding of the embryo's anatomical evolution.

The proposed development of a Virtual Reality system that contains 3D models of the human embryo as well as additional educational content is different from previous work in that the models of the embryos will be constructed by using computer graphic techniques rather than reconstructing microscopic or magnetic resonance images, as well as including a user acceptance study into the research.

\section{Rationale}

Previous literature elaborates on some of the difficulties arising from using traditional learning methods in the field of anatomy in general (Falah et al., 2013; Falah et al., 2015), and embryology as one of its subdivisions (Carlson, 2002; Moraes \& Pereira, 2010). Several research papers also demonstrate how novel technologies such as VR have the potential to aid the educational process in a variety of fields, including medical education, in particular basic medical sciences such as anatomy and embryology (Dimitropoulos et al. 2007; Falah et al., 2013; Falah et al., 2014; Ginani et al. 2012). However there is a scarcity of research evaluating the acceptance of users of such new technology into their learning process, and their willingness to use it on a regular basis as an integral part of the curriculum. This information is crucial prior to the implementation of any new learning method (Sánchez et al., 2000; Dillon, 2001; Venkatesh et al., 2002), and can be assessed by formulating the following three research objectives, that this paper aims to answer:

- To clarify the nature of the current educational modalities used to deliver the embryology course material, and how well the current learning and teaching methods represent the embryos actual structure, and facilitate the learning process.

- To identify any shortfalls of the currently used methods.

- To establish whether the addition of VR technology to the existing modalities has the potential to overcome these shortfalls, and to enhance the students learning experience.

\section{Conceptual Framework}

A model defending the metaphorical design of educational VR systems was proposed by Sanchez et al., 2000, based on Lakoff and Johnson's theory of cognition. This model can be used to evaluate and justify the introduction of VR technology as an educational tool in any curriculum. Alfalah et al., 2017, in their research to evaluate perceptions of students towards the adoption of VR as a learning aid in IT education, adopted this. In this research this framework will be adapted from Alfalah et al., 2017, to apply it to the field of embryology education. Figure 1 shows the adapted model as follows: 


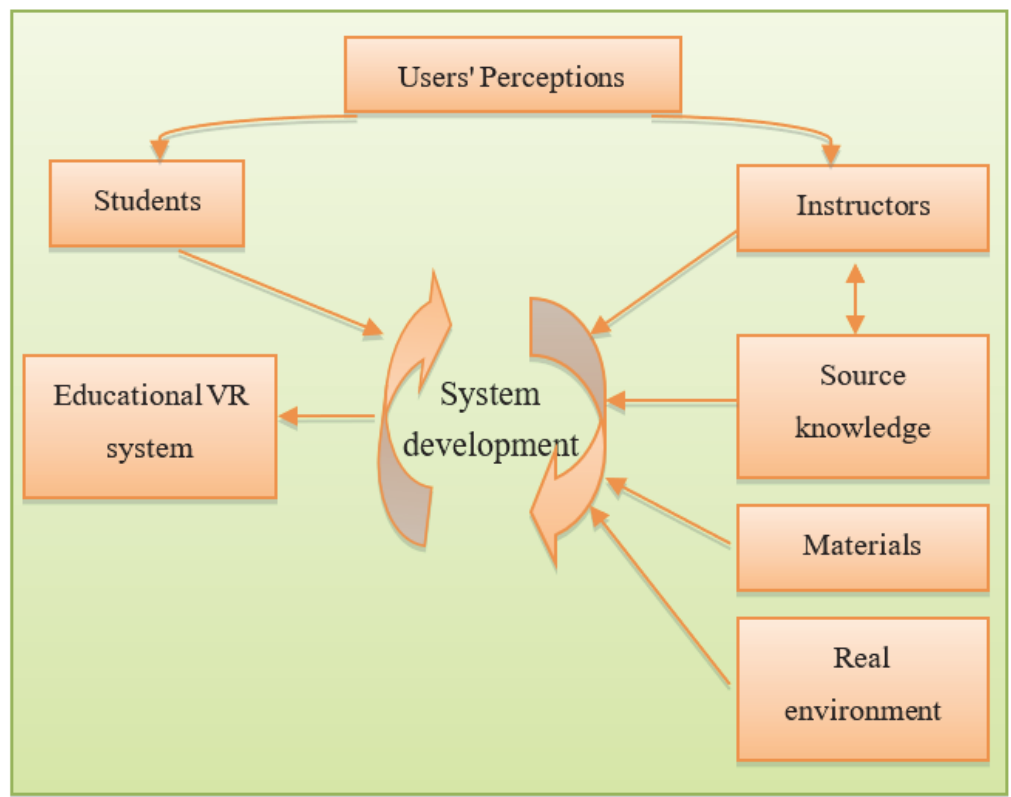

Figure 1. Educational VR system model (Adapted from (Alfalah, 2017))

- User perceptions - The perceptions of the system users toward adopting VR technology as a learning medium: the first group of users "Students", and the second group of users "Instructors".

- Source knowledge - Represents all the concepts, skills, to be learnt by the student.

- Real Environment - The setting in which teaching takes place.

- Materials - Represent didactic materials and information related to the subject.

- System Development - The actual implementation of the VR system.

- Educational VR system - VR system for IT education as a learning medium.

In this research, the focus will be mainly on the first group of users, namely the students, as the quantitative data is derived from the questionnaire distributed amongst them, whereas qualitative data from interviews and the focus group is obtained from groups, students and instructors.

\section{Methodology}

This research was concerned with evaluating the currently used methods that are employed by second year medical and dentistry students to learn the embryology course material that is taught as part of the anatomy curriculum. Furthermore, it was important to identify certain hindrances faced by the students as a result of resorting to traditional educational tools, and what students would consider to be a useful addition to the current system, which they are willing to use in their learning process. Eliciting these points aims to lay the foundation for justifying the development of a Virtual reality tool to enhance learning the embryology material and incorporating this into the educational program.

\subsection{Population}

This paper present a case study performed in the Faculty of Medicine, University of Jordan. Interviews were conducted with a focus group consisting of 15 individuals (members of the teaching staff and students who are current taking the embryology course as part of their basic sciences curriculum), examining the current methods for delivering the course material to the students, the difficulties faced by the students while studying the material and attempting to understand and visualize the events that occur during embryogenesis, and their views on the use of IT during their revision.

This resulted in the design of a questionnaire consisting of 20 questions, aiming to examine the current teaching methods employed for delivering the course material, as well as the degree of student satisfaction with these methods. Furthermore, the current system was explored for any potential shortfalls that could be addressed by enhancing the available teaching methods with novel technologies, such as virtual reality, and the degree of acceptance of the users of such changes to their way of learning, to support the proposal of a VR system as a 
teaching and learning aid.

After the design of the questionnaire, it was distributed to a sample consisting of 120 medical and dentistry students in their second year of undergraduate studies in the University of Jordan, based on the fact that these students were at the time attending embryology lectures as part of their anatomy curriculum, and are therefore aware of the methods used to deliver the course material, their advantages and disadvantages, and any possible difficulties they face during the learning process. The total number of students that completed the questionnaire was 120 .

\subsection{Instrumentation}

The methodology is a mixed method research utilizing qualitative and quantitative methods consisting of interviews conducted with a focus group of 15 members of staff and embryology students, during which the researchers explored the current system used to deliver the embryology course material, to formulate the research questions that the questionnaire aims to answer, followed by the design of a questionnaire distributed as a hardcopy to all students attending the embryology course at the time of the research. The questionnaire included 16 questions (divided into the following categories: demographics (question 1), current methods of learning and assessment of the embryology material (questions 2-4, Table 1), students' perceptions towards the current methods (questions 511 , Table 2), and students' perceptions towards the introduction of novel technology into the curriculum (questions 12-16, Table 3).

After identification of the participants' gender (question 1), the first part of the questionnaire aims to gather information about the nature of learning methods available in the faculty that the students' employ to learn the course material (Table 1), the students' preferences regarding assessment methods for the course material, and any other extracurricular tools used by the students to learn the material.

Table 1. Questions about current methods of learning and assessment of the embryology material

\begin{tabular}{|c|c|c|c|}
\hline \multicolumn{4}{|c|}{ PART I: learning and assessment methods } \\
\hline Question No. & Question & Answer & \\
\hline 2 & $\begin{array}{l}\text { What is the nature of the learning tools you use to learn } \\
\text { the embryology material }\end{array}$ & Theoretical & Practical \\
\hline 3 & $\begin{array}{l}\text { What assessment method do you prefer for the } \\
\text { embryology examination }\end{array}$ & $\begin{array}{l}\text { Multiple choice } \\
\text { questions }\end{array}$ & $\begin{array}{l}\text { Multiple choice questions } \\
\text { and practical assessment }\end{array}$ \\
\hline 4 & $\begin{array}{l}\text { What extracurricular method do you prefer to aid you in } \\
\text { understanding the course material }\end{array}$ & IT resources & $\begin{array}{l}\text { Non-It } \quad \text { Combination } \\
\text { resources }\end{array}$ \\
\hline
\end{tabular}

The second part evaluates the students views on the current teaching and learning methods (Table 2), with regards to achieving a true representation of the 3D structure of the embryo, enabling adequate visualization of such structure, accessibility and availability, and whether these methods present the material in a way that is easy and quick to understand, prevents distraction, ensures efficient retaining of the learned information, and offers an enjoyable experience.

Table 2. Questions about students' perceptions towards the current methods. Students were asked to rate their responses on a 5-point Likert scale as follows: Never (1), Rarely (2), Sometimes (3), Often (4), Always (5).

\begin{tabular}{ll}
\hline PART II: Current Learning Methods \\
\hline Question No. & Question \\
\hline 5 & $\begin{array}{l}\text { Do you think the current methods used to deliver the course material provide a true } \\
\text { representation of the } 3 D \text { structure of the embryo }\end{array}$ \\
6 & $\begin{array}{l}\text { Do you think the current methods used to deliver the course material provide good } \\
\text { visualization of the } 3 D \text { structure of the embryo } \\
\text { Are the current methods for learning embryology accessible and available to you when } \\
\text { needed (or can you only access them at certain times) }\end{array}$ \\
8 & $\begin{array}{l}\text { Are the current methods for learning embryology easy and quick to understand } \\
\text { Do you think using traditional learning methods is sufficient to prevent getting distracted } \\
\text { during learning of the embryology material }\end{array}$ \\
10 & Do you efficiently retain the information gain from learning from the current learning \\
& resources (is it easily memorized) \\
11 & Do you find learning the course material enjoyable
\end{tabular}


The third section of the questionnaire enquires about students' practices and perceptions with regard to extracurricular learning aids in general, and particularly those involving IT and VR (Table 3). Students were asked how frequently they resort to extracurricular learning tools, whether they would like to use novel IT application in their learning process, whether they have any previous exposure to VR technology in general, whether they think that it has the potential to enhance the learning experience in the field of embryology, and finally whether a 3D human embryo system would be used by the students if made available to them.

Table 3. Questions about students' perceptions towards the introduction of novel technology into the curriculum. Responses were rated on a 5-point likert scale as follows: Never (1), Rarely (2), Sometimes (3), Often (4), Always (5)

\begin{tabular}{ll}
\hline & PART III: Perceptions regarding extracurricular tools and IT technology \\
\hline Question No. & Question \\
\hline 12 & Do you use extracurricular methods to aid you in understanding the course material \\
13 & Would you like to use modern IT applications to aid in the learning process \\
14 & Do you use Virtual reality technology (educational, gaming, etc.) \\
15 & Do you think Virtual Reality Technique has the potential to enhance the way of giving the \\
& material \\
16 & Do you think a 3 dimensional Embryo system would be usable by the students \\
\hline
\end{tabular}

\section{Data Analysis}

\subsection{Statistical Treatments}

Qualitative analysis of the interview responses was utilized to formulate the three main research questions and to develop the research questionnaire. This was followed by quantitative analysis of the data obtained from the questionnaire using Statistical Package for Social Sciences (SPSS).

\subsection{Standard deviation}

The researcher firstly used the standard deviation for each question to ascertain whether the participants understood the question and answered them accordingly, to measure confidence in statistical conclusions. Table 4 demonstrates the minimum and maximum responses for each question in the questionnaire, as well as the mean and standard deviation for each set of answers. It demonstrates that the standard deviation for all but one question (Q6) is less than 1.

Table 4. Standard deviation for Each Question

\begin{tabular}{llllll}
\hline Questions & N & Minimum & Maximum & Mean & Std. Deviation \\
Q1 Gender & 120 & 1 & 2 & 1.75 & .435 \\
Q2 Course material Preferred & 119 & 1 & 3 & 1.29 & .693 \\
Q3 Assessment Preferred & 120 & 2 & 3 & 2.02 & .129 \\
Q4 Extracurricular tool & 119 & 1 & 3 & 1.42 & .797 \\
Q5 Current modalities & 120 & 1 & 5 & 3.53 & .943 \\
Q6 Current modalities & 120 & 1 & 5 & 3.66 & 1.017 \\
Q7 Current modalities & 120 & 1 & 3 & 1.79 & .809 \\
Q8 Current modalities & 120 & 1 & 5 & 3.66 & .845 \\
Q9 Current modalities & 120 & 1 & 5 & 3.97 & .995 \\
Q10 Current modalities & 120 & 1 & 5 & 3.85 & .976 \\
Q11 Current modalities & 120 & 1 & 5 & 3.97 & .925 \\
Q12 Perceptions toward technology & 120 & 1 & 3 & 2.25 & .781 \\
Q13 Perceptions toward technology & 120 & 1 & 3 & 1.18 & .502 \\
Q14 Perceptions toward technology & 120 & 1 & 3 & 1.64 & .683 \\
Q15 Perceptions toward technology & 120 & 1 & 3 & 1.26 & .615 \\
Q16 Perceptions toward technology & 120 & 1 & 3 & 1.19 & .473 \\
Valid N (listwise) & 118 & & & & \\
\hline
\end{tabular}


Table 5 demonstrates the minimum and maximum responses for each section of the questionnaire, as well as the mean and standard deviation for each set. It demonstrates that the standard deviation for all section is less than 1 .

Table 5. Standard deviation for each section

\begin{tabular}{llllll}
\hline Questions & N & Minimum & Maximum & Mean & $\begin{array}{l}\text { Std. } \\
\text { Deviation }\end{array}$ \\
\hline PART I: Separate analysis (Q2-Q4) & 120 & 1 & 3 & 1.62 & .286 \\
PART II: Favor of traditional methods (Q5-Q11) & 120 & 3 & 4 & 3.59 & .335 \\
PART III: Favor of new system (Q12-Q16) & 120 & 1 & 3 & 1.52 & .295 \\
Valid N (listwise) & 120 & & & & \\
\hline
\end{tabular}

\subsection{Question 1: Sample Demographics}

Table 6 demonstrates the responses to question 1, namely the gender distribution of the 120 students who completed the questionnaire, which shows a female to male ratio of $3: 1$.

Table 6. Sample gender distribution

\begin{tabular}{llllll}
\hline Answer & Frequency & Percent & Valid Percent & Cumulative Percent \\
\hline Valid & Male & 30 & 25.0 & 25.0 & 25.0 \\
& Female & 90 & 75.0 & 75.0 & 100.0 \\
& Total & 120 & 100.0 & 100.0 & \\
\hline
\end{tabular}

\subsection{Part I (Questions 2-4) analysis}

Question 2: Nature of learning tools utilized by students within the faculty

Table 7 shows the percentages of students using theoretical, practical, or a combination of both resources, made available to them within the faculty to learn the embryology material. It reveals that $83.3 \%$ use only theoretical resources, i.e. the lectures provided by the teaching staff, $2.5 \%$ rely only on the use of practical resources such as models and preserved embryos, whereas $13.3 \%$ resort to a combination of both practical and theoretical resources to study the course material. This indicates the majority of students do not utilize practical learning resources in the current system.

Table 7. Nature of learning tools

\begin{tabular}{llllll}
\hline Answers & & Frequency & Percent & Valid Percent & Cumulative Percent \\
\hline Valid & Theoretical & 100 & 83.3 & 84.0 & 84.0 \\
& Practical & 3 & 2.5 & 2.5 & 86.6 \\
& Both (Theo \& Pract) & 16 & 13.3 & 13.4 & 100.0 \\
& Total & 119 & 99.2 & 100.0 & \\
Missing & 0 & 1 & .8 & & \\
Total & 120 & 100.0 & & \\
\hline
\end{tabular}

\section{Question 3: Preferred Assessment Method}

Table 8 demonstrates the students' preferences with regard to the assessment tools they would like to be applied during course examinations. $98.3 \%$ of responses state that they would prefer a combination of practical assessment and multiple choice questions, whereas only $1.7 \%$ prefer the currently used multiple choice question system.

Table 8. Students preferences regarding the nature of assessments

\begin{tabular}{llllll}
\hline Answers & Frequency & Percent & Valid Percent & Cumulative Percent \\
\hline Valid & Both (Essay \& Multiple) & 118 & 98.3 & 98.3 & 98.3 \\
& Multiple Choice & 2 & 1.7 & 1.7 & 100.0 \\
& Total & 120 & 100.0 & 100.0 & \\
\hline
\end{tabular}


Question 4: Extracurricular learning methods:

Table 9 shows the nature of extracurricular resources students prefer to resort to aid them in the understanding of the course material. $77.3 \%$ prefer some form of IT technology, such as the Internet, to aid them in their learning process, whereas $3.4 \%$ prefer non-IT resources such as models in their studies, and $19.3 \%$ prefer a combination of both IT and other resources.

Table 9. Extracurricular methods preferred by students to understand embryology material in addition to textbooks

\begin{tabular}{llllll}
\hline Answers & & Frequency & Percent & Valid Percent & Cumulative Percent \\
\hline Valid & Internet (Youtube, etc). & 92 & 76.7 & 77.3 & 77.3 \\
& Models. & 4 & 3.3 & 3.4 & 80.7 \\
& combination. & 23 & 19.2 & 19.3 & 100.0 \\
& Total & 119 & 99.2 & 100.0 & \\
Missing & 0 & 1 & .8 & & \\
Total & & 120 & 100.0 & & \\
\hline
\end{tabular}

\subsection{Part II (Questions 5-11) Analysis}

Responses of questions in favor of traditional methods of teaching (questions 5 till 11) were found to be in the rejection area, these results could be accepted since the standard deviation is less than one except question 6 .

\subsection{Part III (Questions 12-16) Analysis}

Responses of questions in favor of using new technology (questions 12 till 16) were found in the acceptance area, these results can be accepted since the standard deviation is less than one.

Overall, these results indicate that students are not satisfied with the traditional educational modalities used to deliver the embryology material, and are in favor of implementing new techniques to aid the learning process.

\subsection{Cronbach's Alpha}

Cronbach's alpha after analysis was found to be 0.704 as shown in Table 10 . This implies the reliability level, as the level of internal consistency for our scale with this specific sample is more than $70 \%$. Therefore, we can accept the result and it can be generalized.

Table 10. (A) and (B) Result of Cronbach's alpha

(A) Case Processing Summary

\begin{tabular}{llll}
\hline \multirow{3}{*}{ Cases } & Valid & $\mathrm{N}$ & $\%$ \\
& Excluded $^{\mathrm{a}}$ & 0 & 100.0 \\
& Total & 120 & .0 \\
& & 100.0
\end{tabular}

a. Listwise deletion based on all variables in the procedure.
(B) Reliability Statistics

\begin{tabular}{ll}
\hline Cronbach's Alpha & N of Items \\
.704 & 12 \\
\hline
\end{tabular}

Table 11 shows the result of Cronbach's alpha if we delete one question and the result shows the level of internal consistency its between $65 \%$ - $72 \%$ that means we can accept the result and generalize it. 
Table 11. Cronbach's alpha Item is deleted one - Item-Total Statistics

\begin{tabular}{lllll}
\hline $\begin{array}{l}\text { Question } \\
\text { No. }\end{array}$ & Scale & Mean & ifScale Variance ifCorrected & \multicolumn{2}{c}{ Item-Cronbach's Alpha if } \\
Item Deled & Item Deleted & Total Correlation & Item Deleted \\
\hline Q5 & 29.70 & 21.674 & .044 & .724 \\
Q6 & 28.42 & 17.590 & .521 & .654 \\
Q7 & 28.29 & 17.183 & .520 & .653 \\
Q8 & 30.16 & 20.353 & .219 & .702 \\
Q9 & 28.29 & 18.427 & .477 & .664 \\
Q10 & 27.98 & 17.311 & .519 & .653 \\
Q11 & 28.10 & 17.234 & .545 & .649 \\
Q12 & 27.98 & 17.159 & .598 & .641 \\
Q13 & 30.77 & 21.979 & .079 & .711 \\
Q14 & 30.31 & 21.022 & .178 & .705 \\
Q15 & 30.69 & 22.181 & .007 & .721 \\
Q16 & 30.76 & 21.933 & .101 & .709 \\
\hline
\end{tabular}

\subsection{Correlation}

Table 12 shows the correlation between data and the relation between traditional data and the new proposed technology VR.

The relation between traditional and the new technology is negative, which means the system will achieve its desired goals, since the first group of questions are in the rejection area and the second group of questions are in the acceptance area as abovementioned.

Table 12. Correlation between data and the relation between traditional data and the new proposed technology VR

\begin{tabular}{|c|c|c|c|}
\hline \multicolumn{2}{|l|}{ Correlation } & \multicolumn{2}{|c|}{ Favor of traditional methodsFavor of new system } \\
\hline \multicolumn{3}{|c|}{ Favor of traditional methodsPearson Correlation1 } & -.119 \\
\hline \multicolumn{3}{|c|}{ Sig. (2-tailed) } & .195 \\
\hline \multirow{4}{*}{ Favor of new system } & $\mathrm{N}$ & 120 & 120 \\
\hline & Pearson Correl & 1-.119 & 1 \\
\hline & Sig. (2-tailed) & .195 & \\
\hline & $\mathrm{N}$ & 120 & 120 \\
\hline
\end{tabular}

\section{Discussion and Results}

Research Objective One: To clarify the nature of the current educational modalities used to deliver the embryology course material, and how well the current learning and teaching methods represent the embryos actual structure, and facilitate the learning process.

The focus group discussions and participants' responses to the questionnaire reveal that the embryology course consists mainly of theoretical material, such as textbooks and lectures, and the students are assessed through a written exam consisting largely of multiple-choice questions. There are no significant practical session to support the delivery of the course material, and no practical assessment of the gained knowledge. This is consistent with the general trend of embryology teaching as described by Carlson (2002).

With regard to available resources at the faculty, only a minority of students access practical resources, whereas the majority studies from textbooks and lecture material.

The responses to the questionnaire reveal however, that students tend to resort to extracurricular methods to aid them in learning and understanding the course material. More than three quarters of the students prefer IT resources, such as YouTube videos to aid them in learning the course material, and the rest prefer non IT learning aids, such as models and others, or a combination of both. This confirms what previous studies suggest regarding students attitudes towards using novel technologies in the learning process (Alfalah et al., 2017; Kron et al., 2010).

Research Objective Two: To identify any shortfalls of the currently used methods

The focus group discussion highlighted shortfalls regarding the current methods of learning embryology amongst the students, which were confirmed from the students' responses to the questionnaire. Students feel that the current 
resources provided to learn the course material does not provide a true representation of the embryo's threedimensional structure. The responses also imply that the current learning methods cannot achieve proper 3D visualization of the embryonic structure.

Furthermore, students feel that the way the material was presented was not sufficient to prevent distraction during learning. They also felt that they were not able to efficiently retain the material when studying using the traditional learning tools. In addition to the previous points, students did not consider learning the course material enjoyable.

Current methods for learning embryology were deemed to be accessible and available to the students when needed, however students did not consider these methods easy and quick to understand.

Similar observations have been made in previous studies in embryology and other fields of medical education such as anatomy (Moraes, \& Pereira, 2010; Falah et al., 2015; Falah et al., 2013), suggesting that traditional learning tools are not sufficient to keep up with the fast pace of modern learning, and students' aspirations.

Research Objective Three: To establish whether the addition of VR technology to the existing modalities has the potential to overcome these shortfalls, and to enhance the students learning experience

Students tend to resort to extracurricular resources to aid them in the understanding of the course material, and they did express a willingness to use modern IT technologies in the learning process.

A significant number of students have had previous exposure to Virtual Reality technology in a number of fields such as education or entertainment, and students feel that this technology has the potential to enhance the way of delivering the course material, and that a VR system would be usable by the students if it was made available to them.

This provides a favorable basis to justify developing a virtual reality system to provide a learning tool for embryology students, as user acceptance is the first step in successfully introducing a new technology into any domain (Alfalah, 2018; Alfalah et al., 2017; Sánchez et al., 2000; Dillon, 2001; Venkatesh et al., 2002).

\section{Limitation}

The aim of this study is to examine the current status of embryology learning methods, their shortfalls, and whether the introduction of VR technology into the curriculum has the potential to enhance the learning process. However, this study was carried out on one sample of students within one university, which implies that results should be interpreted with caution when it comes to generalizing its results until results from other educational establishments where similar courses are taught become available.

\section{Conclusion}

There is plenty of evidence in the literature that virtual reality technology has the potential to enhance the educational process by aiding 3D understanding and visualization, reducing the effort to memorize the material and improving information retention, all while offering an enjoyable learning experience.

This research was designed to answer three research questions focusing on clarifying the current system used to deliver the embryology course material, exposing some of its shortfalls, and explore the views of students regarding the introduction of Virtual Reality technology into the learning process, through a mixed method research utilizing interviews and a questionnaire.

The results showed that the theoretical nature of the course and its assessments, results in a number of issues faced by the students, resulting in difficulties with understanding, visualizing and retaining the course material. A large number of students also report lack of enjoyment while studying the subject of embryology.

Students tend to resort to extracurricular learning aids, mainly IT resources such as the Internet, to overcome these shortfalls.

This research demonstrates that students are willing to accept the introduction of novel technology, namely Virtual Reality, into the embryology curriculum, and feel that this will have the potential to enhance the delivery of the material in a positive manner. This provides a favorable basis to support the development of a Virtual Reality embryology system as a tool to be used alongside the current teaching methods, to enhance the learning process and bridge some of the gaps faced by the current system.

\section{Recommendations}

\section{Recommendations for further study}

The results of this study are promising with regard to the perceived benefits of adding VR technology as a learning aid for embryology courses. However, this subject requires further evaluation in a number of dimensions to be 
able to generalize these results:

1. Other studies with similar objectives should be performed on samples of students in other universities and geographical areas to compare results.

2. A similar study should be conducted to evaluate teaching staff's attitudes and perceptions towards VR technology as a learning medium for embryology.

3. More research is needed to assess the practical implications and training needs that accompany the introduction of VR technology into the course.

4. The next step in this research should focus on the actual development of a Virtual Reality embryology learning system, and to assess its usefulness by performing a comparative evaluation between the new system and the currently used learning modalities.

Recommendations for practice

General recommendations to more effectively enable VR technology integration into the learning process in the field of embryology:

1. Educate students and teaching staff about benefits of adding VR technology to the available learning aids, through awareness sessions held prior to implementation.

2. Train both students and teacher in the use of VR technology through dedicated training programs to achieve the desired benefits of adding such technology, and allow sufficient timeslots within the students' and staff's schedules to facilitate this.

3. The technical requirements for establishing a dedicated facility for implementing VR technology should be made available according to a strategic plan clarifying the requirements and timeframe to introduce this new technology.

\section{References}

Alfalah, S. F. M., \& Educ. Inf. Technol. (2018). https://doi.org/10.1007/s10639-018-9734-2

Alfalah, S., Falah, J., Alfalah, T., Elfalah, M., \& Falah, O. (2017). Perceptions toward Adopting Virtual Reality as a Learning Aid in Information Technology. World Academy of Science, Engineering and Technology, International Journal of Computer, Electrical, Automation, Control and Information Engineering, 11(4), 457461.

Carlson, B. M. (2002). Embryology in the medical curriculum. The Anatomical Record, 269(2), 89-98.

Dillon, A. (2001). User acceptance of information technology. In Encyclopedia of human factors and ergonomics. London: Taylor and Francis.

Dimitropoulos, K., Manitsaris, A., \& Mavridis, I. (2007). Building Virtual Reality Environments for Distance Education on the Web: A Case Study in. In Medical Education, " Computer Journal of International Journal of Social Sciences.

Dimitropoulos, K., Manitsaris, A., \& Mavridis, I. (2008). Building virtual reality environments for distance education on the web: A case study in medical education. International Journal of Social Sciences, 2(1), 6270.

Dorland, W. (2007). Dorland's medical dictionary for health consumers. Saunders, an imprint of Elsevier.

Drake, R. L., McBride, J. M., Lachman, N., \& Pawlina, W. (2009). Medical education in the anatomical sciences: The winds of change continue to blow. Anatomical sciences education, 2(6), 253-259.

Drake, R. L., Lowrie, D. J., \& Prewitt, C. M. (2002). Survey of gross anatomy, microscopic anatomy, neuroscience, and embryology courses in medical school curricula in the United States. The Anatomical Record, 269(2), $118-122$.

Falah, J., Charissis, V., Khan, S., Chan, W., Alfalah, S. F., \& Harrison, D. K. (2015). Development and evaluation of virtual reality medical training system for anatomy education. In Intelligent Systems in Science and Information 2014 (pp. 369-383). Springer, Cham.

Falah, J., Harrison, D. K., Charissis, V., \& Wood, B. M. (2013). July. The characterisation of a virtual reality system to improve the quality and to reduce the gap between information technology and medical education. In International Conference on Virtual, Augmented and Mixed Reality (pp. 122-131). Springer, Berlin, Heidelberg. 
Falah, J., Khan, S., Alfalah, T., Alfalah, S. F., Chan, W., Harrison, D. K., \& Charissis, V. (2014). August. Virtual Reality medical training system for anatomy education. In Science and Information Conference (SAI), 2014 (pp. 752-758). IEEE.

Ginani, F., Gadelha, R., \& Augusto, G. (2012). Use of clinical cases in a virtual learning environment as an approach to teaching Human Embryology. Int. J. Morphol, 30(4), pp.1395-1398Klimoski, R., \& Palmer, S. (1993). The ADA and the hiring process in organizations. Consulting Psychology Journal: Practice and Research, 45(2), 10-36. https://doi.org/10.1037/1061-4087.45.2.10

Kron, F. W., Gjerde, C. L., Sen, A., \& Fetters, M. D. (2010). Medical student attitudes toward video games and related new media technologies in medical education. BMC medical education, 10(1), 50.

Moraes, S. G., \& Pereira, L. A. V. (2010). A multimedia approach for teaching human embryology: Development and evaluation of a methodology. Annals of Anatomy-Anatomischer Anzeiger, 192(6), 388-395.

Nicholson, D. T., Chalk, C., Funnell, W. R. J., \& Daniel, S. J. (2006). Can virtual reality improve anatomy education? A randomised controlled study of a computer generated three dimensional anatomical ear model. Medical education, 40(11), 1081-1087.

Patel, K. M., \& Moxham, B. J. (2008). The relationships between learning outcomes and methods of teaching anatomy as perceived by professional anatomists. Clinical anatomy, 21(2), 182-189.

Richardson, A., Hazzard, M., Challman, S. D., Morgenstein, A. M., \& Brueckner, J. K. (2011). A "Second Life" for gross anatomy: Applications for multiuser virtual environments in teaching the anatomical sciences. Anatomical Sciences Education, 4(1), 39-43.

Sánchez, Á., Barreiro, J. M., \& Maojo, V. (2000). Design of virtual reality systems for education: a cognitive approach. Education and Information Technologies, 5(4), 345-362.

Scott, K. M., Charles, A. R., \& Holland, A. J. (2013). Clinical embryology teaching: is it relevant anymore? ANZ Journal of Surgery, 83(10), 709-712.

Venkatesh, V., Speier, C., \& Morris, M. G. (2002). User acceptance enablers in individual decision making about technology: Toward an integrated model. Decision sciences, 33(2), 297-316.

Yamada, S., Uwabe, C., Nakatsu, K. T., Minekura, Y., Iwakura, M., Motoki, T., ... Mizuta, S. (2006). Graphic and movie illustrations of human prenatal development and their application to embryological education based on the human embryo specimens in the Kyoto collection. Developmental Dynamics, 235(2), 468-477.

\section{Copyrights}

Copyright for this article is retained by the author(s), with first publication rights granted to the journal.

This is an open-access article distributed under the terms and conditions of the Creative Commons Attribution license (http://creativecommons.org/licenses/by/4.0/). 